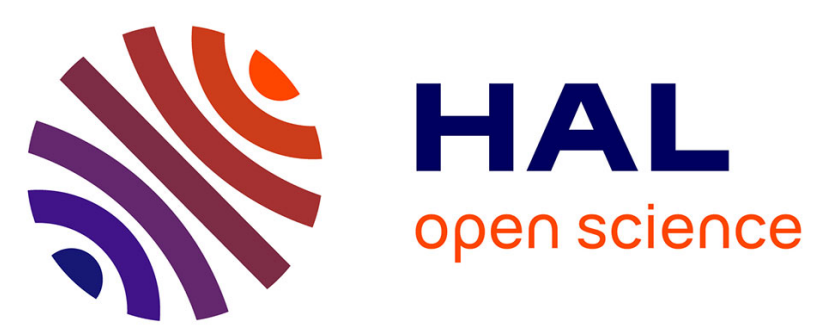

\title{
GRL: A Specification Language for Globally Asynchronous Locally Synchronous Systems
}

Fatma Jebali, Frédéric Lang, Radu Mateescu

\section{To cite this version:}

Fatma Jebali, Frédéric Lang, Radu Mateescu. GRL: A Specification Language for Globally Asynchronous Locally Synchronous Systems. Proceedings of the 16th International Conference on Formal Engineering Methods (ICFEM'14), Nov 2014, Luxembourg, Luxembourg. pp.219-234, 10.1007/9783-319-11737-9_15. hal-01082348

\section{HAL Id: hal-01082348 \\ https://inria.hal.science/hal-01082348}

Submitted on 8 Feb 2016

HAL is a multi-disciplinary open access archive for the deposit and dissemination of scientific research documents, whether they are published or not. The documents may come from teaching and research institutions in France or abroad, or from public or private research centers.
L'archive ouverte pluridisciplinaire HAL, est destinée au dépôt et à la diffusion de documents scientifiques de niveau recherche, publiés ou non, émanant des établissements d'enseignement et de recherche français ou étrangers, des laboratoires publics ou privés. 


\title{
GRL: a Specification Language for Globally Asynchronous Locally Synchronous Systems ${ }^{\star}$
}

\author{
Fatma Jebali, Frédéric Lang, and Radu Mateescu \\ Inria \\ Univ. Grenoble Alpes, LIG, F-38000 Grenoble, France \\ CNRS, LIG, F-38000 Grenoble, France
}

\begin{abstract}
A GALS (Globally Asynchronous, Locally Synchronous) system consists of several synchronous subsystems that evolve concurrently and interact with each other asynchronously. Most formalisms and design tools support either the synchronous paradigm or the asynchronous paradigm but rarely combine both, which requires an intricate modeling of GALS systems. In this paper, we present a new language, called GRL (GALS Representation Language) designed to model GALS systems in an abstract and versatile manner for the purpose of formal verification. GRL has formal semantics combining the synchronous reactive model underlying dataflow languages and the asynchronous concurrent model underlying process algebras. We present the basic concepts and the main constructs of the language, together with an illustrative example.
\end{abstract}

\section{Introduction}

Computer science has led to new generations of heterogeneous systems called GALS (Globally Asynchronous, Locally Synchronous). A GALS system is composed of several synchronous subsystems, executing and interacting in asynchronous concurrency: no assumption is made, neither on the relative frequency of each subsystem, nor on the communication delays between subsystems. Each subsystem is composed of several components running together synchronously, all governed by a single clock and encompassing the zero-delay assumption: computations and communications between components are instantaneous (these are called the synchronous assumptions). As such, GALS systems involve a high degree of synchronous and asynchronous concurrency (introducing nondeterminism), which requires tedious effort to design and debug. Formal modeling and verification is then a crucial part in the design process of such usually safetycritical systems.

Many different approaches have been proposed for GALS modeling and verification. Some propose to model GALS systems in synchronous frameworks (such as Signal [26]) directly or to extend synchronous languages with an asynchronous layer (Multiclock Esterel [4], CRSM [29]). Such representations are well-suited

\footnotetext{
* This work was partly funded by the French Fonds unique interministériel (FUI), Pôle Minalogic (project "Bluesky for I-Automation").
} 
to hardware-based subsystems distributed on one single hardware platform, each with its own clock.

Other approaches, conversely, extend asynchronous languages to incorporate synchronous features. The most common approach is to surround each locally synchronous subsystem by an asynchronous wrapper, which provides an asynchronous interface to other subsystems. This way, GALS systems can be modeled and verified in asynchronous frameworks. In [9], Signal modules are translated to Promela, the input language of the SPIN model checker [20]. In [13], Sam synchronous programs are represented by Mealy functions without internal state and are encapsulated into wrappers modeled in LNT [7], a language of asynchronous concurrent processes inheriting process algebraic concepts and extended with data and the control structures of classical algorithmic programming. LNT is equipped with the CADP verification toolbox [11], which comprises tools for visual checking, model checking, and equivalence checking. Previously, LNT had been used successfully for the analysis of other GALS systems $[8,12,22]$.

All the aforementioned approaches adopt specific techniques of a specific paradigm (synchronous or asynchronous) to accommodate GALS systems. On the one hand, synchronous frameworks are deterministic by nature, and not appropriate to model asynchrony. On the other hand, asynchrony and nondeterminism are granted for free in asynchronous frameworks, but those lack built-in constructs dedicated to synchronous components, which guarantee that system models fulfill the synchronous assumptions.

Moreover, most existing approaches depend strongly on the considered application field (e.g., distributed control systems [31], FPGA and ASIC digital designs [6,28], or networks on chip), the target platform (e.g., software [24], hardware [9], or heterogeneous), and the preferred specification methods (e.g., based on Petri nets [27], automata [16], process algebras [13]). This narrows down the range of systems that can be addressed. On the other hand, the surge in complexity of GALS systems forces designers to tackle (among others) design concepts, synchronous and asynchronous computations, deterministic and nondeterministic behaviour, and verification approaches, which makes the design of such systems increasingly challenging.

To circumvent this complexity, an appealing trend has been to design new languages dedicated to GALS system modeling $[3,24,5]$, which enforce the assumptions of the GALS paradigm. In this paper, we propose GRL (GALS Representation Language), a new specification language with textual syntax and formal semantics, targeting systems consisting of a network of distributed synchronous systems (called blocks) that interact with their environments and exchange data asynchronously via communication mediums. The design of GRL has originally been driven by the need of general-purpose, designer-friendly, and formal representation of GALS systems suitable for efficient verification.

Our approach draws mainly from two semantic foundations. As regards synchrony, GRL holds a dataflow-oriented model based on the block-diagram model, widely used in industry: synchronous components are modeled by blocks connected together hierarchically to build higher-level blocks. Therefore, the GRL 
synchronous model inherits from the simplicity and modularity of the blockdiagram model. As regards asynchrony, GRL was inspired by process algebras, and more particularly by LNT: blocks exchange data by (implicit) rendezvous synchronization with communication mediums connected to other blocks, and the interactions between blocks and their environments work similarly. Thereby, GRL leverages process algebra expressiveness, versatility, and verification efficiency, with a specialization to the GALS paradigm.

GRL was designed with several concerns in mind. First, it provides a sufficiently high abstraction level to fit a wide range of applications, independently from both the target platform (hardware, software, or heterogeneous), the architecture (single or distributed platforms), and the application domain.

Second, GRL is aimed at being a pivot language between industrial design tools (in particular those based on function block diagrams for the synchronous part) and verification tools for both synchronous and asynchronous systems, which guarantee system reliability and correctness. This way, we hope that formal verification methods - claimed traditionally to require high level expertise in theoretical issues - are easier to learn by industrial users, without requiring companies to shift from their actual tools and languages to entirely new production approaches. Indeed, although some approaches seem efficient [15], the high cost of such a shift makes it unlikely to happen in the near future.

Last but not least, GRL is intended to have a user-friendly syntax as it does not require users to have solid background in neither synchronous programming (e.g., clocks are not modeled explicitly), asynchronous concurrent programming (e.g., parallel composition and synchronization), nor formal verification methods. All features are smoothly and tightly integrated to form a language with homogeneous syntax and semantics.

In this paper, we introduce GRL as a first step towards fully-automated verification of GALS systems. It is organized as follows. Section 2 presents some related work. Section 3 presents the language, its formal semantics, and the current status of software tools. Section 4 gives an illustrative GRL model of an aircraft flight control system used in the avionics industry. Finally, Section 5 summarizes the paper and indicates directions for future work.

\section{Related work}

In this section, we review the languages combining synchronous and asynchronous features. CRP [3] combines the Esterel [2] synchronous language and the CSP [19] asynchronous language. Despite its mathematical elegance, CRP is still rarely used in industry since it requires the user to have expertise in both Esterel and CSP. Such expertise is not required for GRL, which was designed to facilitate industrial GALS design. A language close to CRP is SystemJ [24], which extends Java with Esterel-like synchronous model and CSP-like asynchronous model. SystemJ allows efficient code to be generated automatically. However, it lacks rigorous support for fully-automated formal verification and is not suitable for systems with limited resources because of its reliance on Java virtual 
machine as target. To the contrary, GRL is intended to be general-purpose and verification-oriented. Action Language [5] is a state-based approach, which aims at bridging the gap between high specification languages (Statecharts [17], SCR [18], and RSML [23]) and the SPIN model checker. A key difference between this approach and ours is that Action Language adopts a low-level condition/action model whereas GRL is equipped with high-level control structures making GRL models clearer and more structured.

\section{The GRL Language}

The syntax and semantics of GRL are formally described in a research report [21] (76 pages). In this section, we present them briefly and informally. Figure 1 is a simplified presentation ${ }^{1}$ described in EBNF (Extended Backus-Naur Form), where square brackets denote optional syntactic parts and vertical bars denote alternatives. The symbols $K, X$, and $E$ denote respectively literal constants, variables, and expressions (built upon constants, variables, and function applications). The symbols $S, B, N, M, T$, and $f$ denote respectively system, block, environment, medium, type, and record field identifiers.

\subsection{Overview}

GRL specifications are structured in modules, called programs. Each program can import other programs, which promotes code organization and reuse. A GRL program contains the following constructs:

1. types, ranging from predefined types (such as Booleans and naturals) to user-defined types (such as arrays and record types),

2. named constants, visible by all other constructs,

3. blocks, representing the synchronous components,

4. mediums and environments, representing respectively communication mediums and physical or logical constraints on block inputs, and

5. systems, representing the composition and interactions of blocks, environments, and mediums.

In the sequel, these five constructs are called entities, and blocks, environments, and mediums are called actors.

As regards synchronous behaviours, blocks are the synchronous composition of one or several subblocks, all governed by the clock of the highest level block. A block performs a sequence of discrete deterministic steps and preserves an internal state, hereafter called memory. At each step (each cycle of the clock), it consumes a set of inputs, computes a reaction instantaneously, produces a set of outputs, and updates its memory. Within one block (i.e., at actor level), connections between subblocks are carried out using parameters in modes "in" (input) and "out" (output). Every output parameter can be connected to several

\footnotetext{
${ }^{1} 70$ EBNF productions were necessary to present the full language in [21]
} 


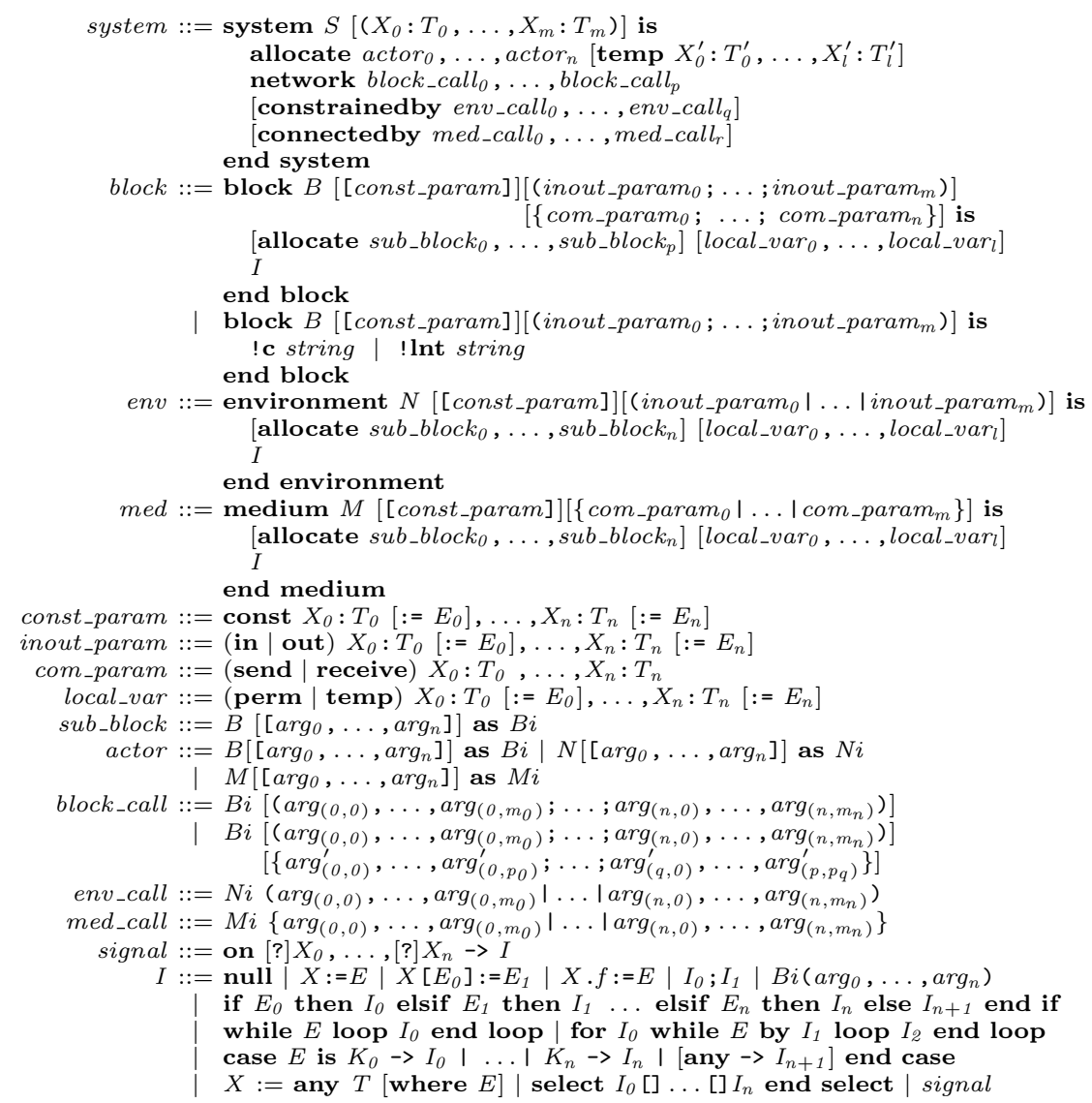

Fig. 1: The syntax of GRL (excerpts)

input parameters of different blocks; however, an input parameter can be connected to only one output parameter of another block. Such connections describe synchronous communication by instantaneous broadcasting.

As regards asynchronous behaviours, blocks are composed, together with environments and mediums, within systems to form networks of distributed connected synchronous subsystems. Within a GRL system (i.e., at system level), the separate blocks execute asynchronously, i.e., each block evolves cyclically at its own frequency (blocks have independent clocks). Blocks interact with each other across mediums, which allows separate blocks to be loosely coupled so that communication is performed asynchronously (i.e., takes an arbitrary amount of time). Connections between blocks and mediums are carried out using parameters in modes "receive" and "send". Receive parameters of mediums can be connected to send parameters of blocks, and conversely. Such connections describe synchronisation and communication by message-passing rendezvous be- 


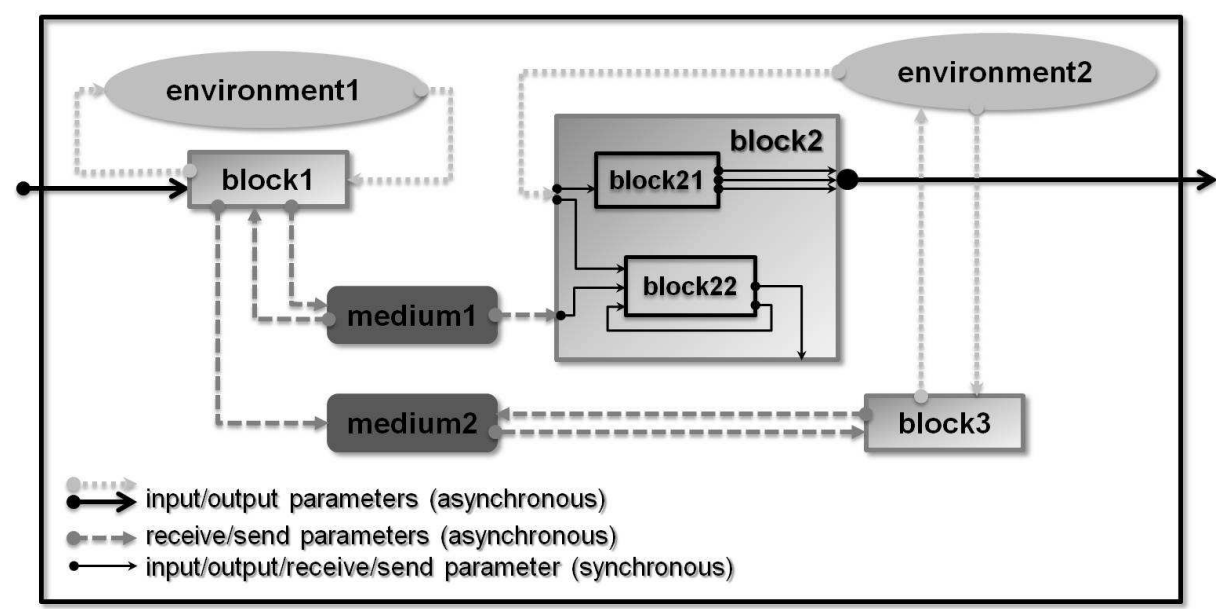

Fig. 2: Schematic representation of a GRL system

tween blocks and mediums. Mediums may exhibit nondeterministic behaviour, a key feature for asynchronous systems modeling and compositional specification; this provides descriptions with accuracy and high abstraction capability.

Blocks behaviour can also be constraint-driven by a collection of user-defined environments. In essence, environments exhibit a similar behaviour as mediums, except that their connections to blocks are carried out using modes "in" and "out". They have been introduced in GRL to explicitly separate the specification of communication mediums from external constraints imposed by the environment; this contributes to provide more comfort and insight about the system composition. With such a composition, we seek enhanced user-convenience to smoothly and tightly tailor complex network topologies, environment requirements and constraints, as well as communication protocols.

As an example, GRL can be used to model the network topology depicted in Figure 2: block1 is constrained by env1 and communicates with block2 and block3 respectively across med1 and med2; block2 and block3 are both constrained by env2; block2 is the synchronous composition of two subblocks.

\subsection{Block}

Blocks, defined by the non-terminal block in Figure 1, are the central building unit of the language. At actor level, a block is defined by the following elements:

- a list "const_param" of typed constant parameters (read-only variables).

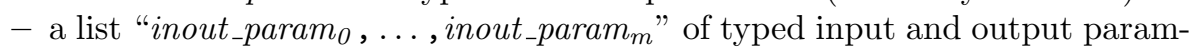
eters preceded by their mode ("in" or "out").

- a list "local_var,$\ldots$, local_var ${ }_{l}$ " of local variables. Temporary variables are declared using the keyword "temp"; their values are lost when the current block execution terminates. Permanent variables are declared using the 
keyword "perm"; their values are kept until the next execution cycle. The memory of the block is the list of values assigned to its permanent variables. - a list "sub_block $k_{0}, \ldots, s_{\text {ob_block}}$ " of subblock allocations, which enables subblock instances to be created, each maintaining its own memory. This concept is inherited from the block-diagram model, which is similar to, but simpler than, the class-instance paradigm in object-oriented languages.

- a body " $I$ " expressed as a deterministic statement defined by combination of high-level control structures (bounded loops, if-then-else, sequential composition, etc.) and synchronous subblock invocations. The scheduling of subblock executions is inherently specified by the order in which the subblocks are invoked, using the sequential composition operator ";". Nondeterministic statements such as " $X:=$ any $T$ where $E$ " and "select" (arbitrary choice of one statement among a set) are forbidden within block bodies.

Alternatively, blocks can be specified in an external language. Their body consists of a pragma denoting the language in which the external function implementing the block is written, followed by the name of the function in the external code. So far, the supported external languages are C and LNT: an external function identifier in C (resp. LNT) is preceded by the pragma "! c" (resp. "!lnt"). Although C external blocks provide more flexibility for the user, they should be defined to comply with the GRL block semantics (in particular, side effects in external $\mathrm{C}$ code are prohibited to enable model checking). LNT external blocks, however, have formal semantics and can thus be used safely.

In a block invocation, actual parameters have different forms according to their modes. A question mark precedes both output and send actual parameters, meaning that the parameter will have a value assigned when returning from the block. An underscore ("_") is used for unconnected parameters (i.e., unused inputs or outputs). An output parameter $X_{i}$ declared in "out $X_{0}: T_{0}, \ldots, X_{n}: T_{n}$ " of a block $B i$ and an input parameter $Y_{j}$ declared in "in $Y_{0}: T_{0}, \ldots, Y_{m}: T_{m}$ " of a block $B i^{\prime}$ can thus be connected synchronously using a variable " $Z: T_{i}$ " by passing "? $Z$ " to $B i$ and " $Z$ " to $B i^{\prime}$ in a subsequent invocation.

Additional elements can be used to define a block that can only be invoked at system level, namely lists "com_paramo,$\ldots$, com_param $_{n}$ " of typed receive and send parameters preceded by their mode "receive" or "send". They enable blocks to interact asynchronously within a network of blocks via mediums. Such a block cannot be allocated nor invoked inside another actor since communication between blocks within actors is necessarily synchronous. At system level, actual input parameters of blocks can have the additional form "any $T$ ", meaning that an arbitrary value of type $T$ is passed as input to the block.

The behaviour of a block is the following. In each cycle of its clock, (1) the block consumes data received over input and receive parameters, (2) the block computes by executing its body, then (3) the block produces data sent over output and send parameters. During computation, its memory is assigned the updated values of permanent variables so as to keep them stored up to the next cycle. As usual in the synchronous paradigm, all these steps are performed in zero-delay, i.e., instantaneously and atomically. 


\subsection{Medium}

Mediums, defined by the non-terminal med in Figure 1, are dedicated to the modeling of communications and asynchronous interactions within a network of synchronous blocks. A medium is defined by the following elements:

- a list "const_param" of constant parameters.

- a list "com_param,$\ldots$, com_param $_{m}$ " of send and receive parameters.

- a list "sub_block $k_{0}, \ldots$, sub_block ${ }_{p}$ " of subblock allocations enabling blocks to be used in mediums in the same way as functions in programming languages.

- a list "local_var,$\ldots$, local_varl" of local (temporary and permanent) variable declarations.

- a body "I" expressed as a statement (not necessarily deterministic) defined as a combination of high-level control structures, subblock compositions, and nondeterministic statements.

A medium sends and receives messages to and from several blocks. When a block wants to send a message to or receive a message from a medium, it triggers the execution of the medium, which we call medium activation. Therefore, the invocation of mediums is demand-driven by different blocks at unpredictable instants. In this respect, mediums are passive actors, whereas blocks are active actors. Each medium is activated during a block execution cycle at most once to send messages to the block, then at most once to receive messages from the block. Since several messages may have to transit via one medium, those messages are grouped in tuples, called channels, all messages of a channel being exchanged within a single block-medium interaction. The channel under consideration is then called activated. The activations of a given medium are thus guided by the separate activations of its channels, as is suggested by the pipe symbol ("I") used to delimit formal and actual channel parameters, each channel activation leading to a separate execution of the medium.

To control medium activations, we introduce signal statements, whose syntax is defined by the non-terminal signal in Figure 1. A signal guards the part of the medium code that needs to be executed upon the activation of a particular channel. When a channel of the form "receive $X_{0}, \ldots, X_{n}$ " is activated, the signal statement "on $X_{0}, \ldots, X_{n} \rightarrow I$ " can be executed and the values of variables $X_{0}, \ldots, X_{n}$ passed to the channel can be read within the statement $I$. When a channel of the form "send $Y_{0}, \ldots, Y_{m}$ " is activated, the signal statement "on ? $Y_{0}, \ldots, ? Y_{m} \rightarrow I$ " can be executed and the statement $I$ must assign values to the variables $Y_{0}, \ldots, Y_{m}$. Static semantics prohibit sequential composition of signals, loop statements containing signals, and nested signals, so that at most one signal is present on each execution path.

Mediums introduce flexibility in system models since they provide an accurate design of complex network topologies (e.g., bus, star, ring, mesh), connection modes (e.g., point-to-point, multi-point), as well as communication protocols. This way, we address a lack identified in existing languages confined to rigid topologies and point-to-point communications between separate synchronous subsystems, such as those based on CSP rendezvous [24,3, 9]. Limita- 
tions of adopting point-to-point communications in GALS models are considered as drastically restrictive to design complex networks of arbitrary topologies [30].

\subsection{Environment}

Since synchronous systems are often recognized to be outside-aware, GRL allows the user to model explicitly, yet abstractly, the behaviour of the environment. There are two major roles the environment can play: impose outside physical and logical requirements that block inputs may undergo, and put constraints on the scheduling of blocks executing in parallel. Additionally, an environment may be local, i.e., connected only to one block, or global, i.e., connected to several blocks at the same time. Environments, defined by the non-terminal env in Figure 1, are syntactically and semantically very similar to mediums, except that send and receive parameters are replaced by input and output parameters.

\subsection{System}

Systems, defined by the non-terminal system in Figure 1, are the top level entities in GRL programs, within which actors are invoked and connected to each other. A system is defined by the following elements:

- a list " $X_{0}: T_{0}, \ldots, X_{m}: T_{m}$ " of parameters, which can be used in actual channels to connect blocks to environments and mediums. They are the visible parameters of the system, observable from the outside world.

- a list "actor ${ }_{0}, \ldots$, actor ${ }_{n}$ " of actor instance declarations.

- a list " $X_{0}^{\prime}: T_{0}^{\prime}, \ldots, X_{l}^{\prime}: T_{l}^{\prime \prime}$ " of temporary variables, which can be used in actual channels to connect blocks to environments and mediums. They are the invisible parameters of the system, not observable from the outside world.

- a list "block_call,$\ldots$, block_call ${ }_{p}$ " of block invocations.

- a list "env_call $, \ldots, e n v_{-} c a l l_{q}$ " of environment invocations that constrain the blocks.

- a list "med_call,$\ldots$, med_call $l_{r}$ " of medium invocations that ensure the traffic inside the network of blocks.

All interactions between actors within a system are built on message-passing synchronisations (rendezvous). Since blocks are the active actors of systems, the scheduling of the whole system is focused around their executions. Blocks execute cyclically, each at its own frequency, and force environments and mediums to perform some operations by sending messages (requesting or providing data) through channels. Environments and mediums, consequently, are passive actors responding to arisen demands from different blocks. At system level, GRL prohibits blocks to be connected directly to each other in order to preserve an independent behaviour of each block and an asynchronous behaviour of the network. Thus, blocks communicate only indirectly across mediums.

Each interaction between two actors is performed through exactly one channel. Namely, an output channel of the form "out $X_{0}: T_{0}, \ldots, X_{n}: T_{n}$ " of an 
actor $A i$ and an input channel of the form "in $Y_{0}: T_{0}, \ldots, Y_{n}: T_{n}$ " of another actor $A i^{\prime}$ can thus be connected using a set of variables " $Z_{0}: T_{0}, \ldots, Z_{n}: T_{n}$ " by passing ? $Z_{0}, \ldots, ? Z_{n}$ to $A i$ and $Z_{0}, \ldots, Z_{n}$ to $A i^{\prime}$, in their respective invocations within a system. Send and receive channels can be connected similarly.

The semantics of a system are the following. Blocks execute arbitrarily often and cyclically. Each time a block begins its execution cycle, all environments and mediums connected respectively to input and receive channels of the block are activated to provide the needed input and receive values. Unconnected input and receive channels are assigned arbitrary values. Then, the block executes its body, and thus updates its output and send channels as well as its memory. Finally, all environments and mediums connected to respectively output or send channels of the block are activated.

The combined execution cycle of a block, and its related environments and mediums is performed instantaneously according to the synchronous assumptions. As a consequence, a block is executed only if all its connected environments and mediums are able to respond to all input, output, receive, and send signals of the block.

\subsection{Formal Semantics}

The semantics of GRL are formally defined in [21]. They consist in 145 rules of static semantics and 24 rules of Plotkin-style structural operational semantics for the dynamic part. In this paper, we only sketch briefly the principles of the dynamic semantics, defined in terms of LTSs (Labelled Transition Systems). An LTS is a quadruple $\left(S, L, \rightarrow, s_{0}\right)$ where $S$ is a set of states, $s_{0} \in S$ is the initial state, $L$ is a set of labels, and $\rightarrow \subseteq S \times L \times S$ is the labelled transition relation.

The memory of an actor, denoted by $\mu$, is a partial function mapping all permanent variables of the actor and its subblocks to their current values. A state $S$ of the system is the union of memories $\mu_{i}$ of all actors composing the system, and the initial state $s_{0}$ maps all permanent variables to their initialization values. Each label has the form $B i\left(a_{0}, \ldots, a_{n}\right)\left\{a_{0}^{\prime}, \ldots, a_{m}^{\prime}\right\}$ with $B i$ a block identifier, $a_{0}, \ldots, a_{n}$ the visible actual parameters of input and output channels, and $a_{0}^{\prime}, \ldots, a_{m}^{\prime}$ the visible actual parameters of receive and send channels. A transition $\mu \stackrel{B i\left(a_{0}, \ldots, a_{n}\right)\left\{a_{0}^{\prime}, \ldots, a_{m}^{\prime}\right\}}{\longrightarrow} \mu^{\prime}$ expresses the combined execution of one cycle of the block instance $B i$, together with its connected environments and mediums. The semantics of the system are obtained by interleaving all possible block executions. Verification (e.g., visual checking, equivalence checking, model checking) can be done by inspection of the LTS.

\subsection{Tools for GRL}

There are currently two software tools for handling GRL models. The first one is a parser for GRL (2000 lines), developed using the SYNTAX and Lotos NT compiler construction technology [10], which performs lexical and syntax analysis, type checking, binding analysis, and variable initialisation analysis of GRL programs. 
The second one, named GRL2LNT (8000 lines), is an automated translator from GRL to LNT. Each block is mapped to an LNT function that takes inputs and produces outputs. Its permanent variables are mapped to inout parameters, i.e., parameters whose values are updated during function invocation. Synchronous block composition is mapped to sequential composition. Additionally, each actor invoked within a system is also mapped to a wrapper process, which contains communication actions to exchange data with its connected actors. The whole system is mapped to the LNT parallel composition of the wrapper processes with appropriate synchronizations of the communication actions.

GRL and GRL2LNT play the role of an intermediate and appropriate layer of abstraction and compositionality to provide generated LNT code with accuracy and conciseness, since scalability of automated model checking is limited. We can take advantage from the CADP toolbox available for LNT to build state spaces and apply visual, equivalence, and model checking techniques.

\section{Example: Flight Control System}

Our aim here is not to present a full case study, but rather to illustrate the main concepts of GRL via a feature rich example: the aircraft Flight Control System (FCS) ${ }^{2}$, whose role is to control the aircraft turning and which is one of the most critical systems inside new generations of Airbus aircraft designs. Subsets of FCS have been studied at different levels of abstraction. In [25], the Flight Guidance System component of the FCS has been studied as a composition of synchronous systems following a single-platform GALS architecture. In [1], control systems have been studied as synchronous systems following a distributed-platforms PALS (Physically Asynchronous, Locally synchronous) architecture. For the sake of simplicity, we model the global behaviour, at a very high level of abstraction, of an FCS containing the following subsystems:

- Flight Control Surfaces adjust and control the aircraft's flight turning. We consider only one aileron (a flap attached to the end of a wing) controller.

- Fly-By-Wire Computers command the movement of the Flight Control Surfaces. We consider only two Fly-By-Wire Computers commanding the position of the aileron, one being used as a backup in case the other fails.

- A Flight Control Data Concentrator schedules the execution of Fly-By-Wire Computers and allows interaction with pilot displays.

The GRL model. The FCS system depicted in Figure 3 (see the GRL code below) consists of four block instances, whose cyclic behaviour is as follows:

- The Ail (for aileron) block instance receives movement requirements from the network, computes the next position of the aileron depending on its current position, then sends it to the network.

\footnotetext{
${ }^{2}$ http://www.skybrary.aero/index.php/Flight_Control_Laws
} 
FlightControlSystem

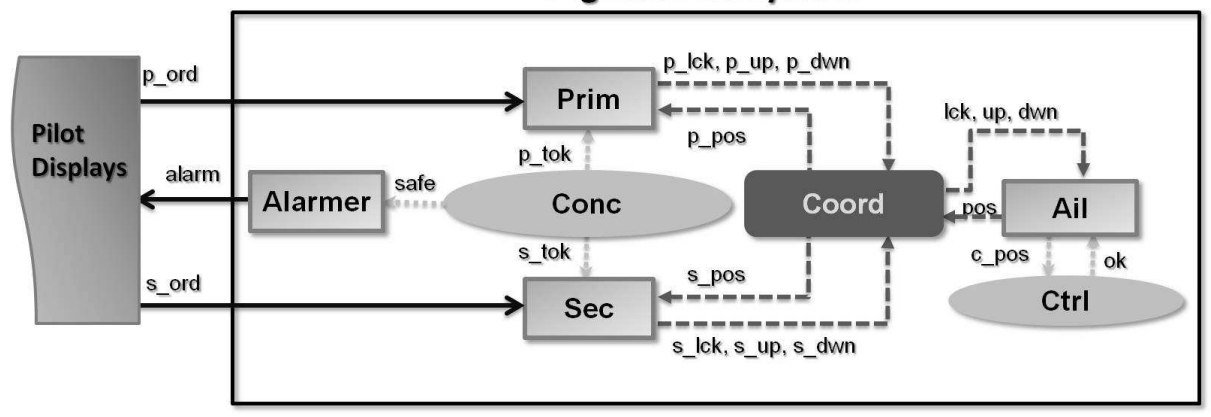

Fig. 3: Architecture of the Flight Control System

- The Prim (for primary) and Sec (for secondary) instances of block FBWComp (for fly-by-wire computer) receive: tokens from the environment indicating whether Prim or Sec should control Ail; an order from the pilot displays; and the current position of the aileron from the network. They compare the two latter values, and then send to the network the decision about whether the aileron should move up, move down, or not move (if the order matches the position).

- The Alarmer block instance checks whether the system is evolving safely by receiving from the environment a message indicating whether Ail is still controlled by either Prim or Sec, then informs the pilot about the safety state of the system.

Communications within the network of blocks are modeled by the medium Coord (for coordinator) as follows. Prim provides Ail with move requirements, then $A i l$ achieves the required computations and sends its new position to Prim. Sec and Ail communicate similarly.

The environment constraints are modeled by two environments. The first environment Conc (for concentrator) ensures that either Prim or Sec, but not both, can control Ail, the priority being given to Prim by activating its token. Conc determines whether Prim is in a safety state (i.e., able to control Ail). Once Prim is not in safety state, it is considered out of order (not alive). Then, Conc blocks the execution of Prim by inactivating its token and gives the control of $\mathrm{Ail}$ to $\mathrm{Sec}$ by activating its token. Once $\mathrm{Sec}$ is not in safety state anymore, which means that neither Prim nor Sec is in safety state, then Ail is considered out of control and Alarmer warns the pilot.

The second environment Ctrl (for controller) constrains the execution of Ail as follows. Ail sends cyclically its new position to Ctrl. Ctrl verifies whether the position is still within a predefined interval, which means that Ail moves smoothly; if not, the execution of $A i l$ is blocked. The GRL specification of the system follows. 


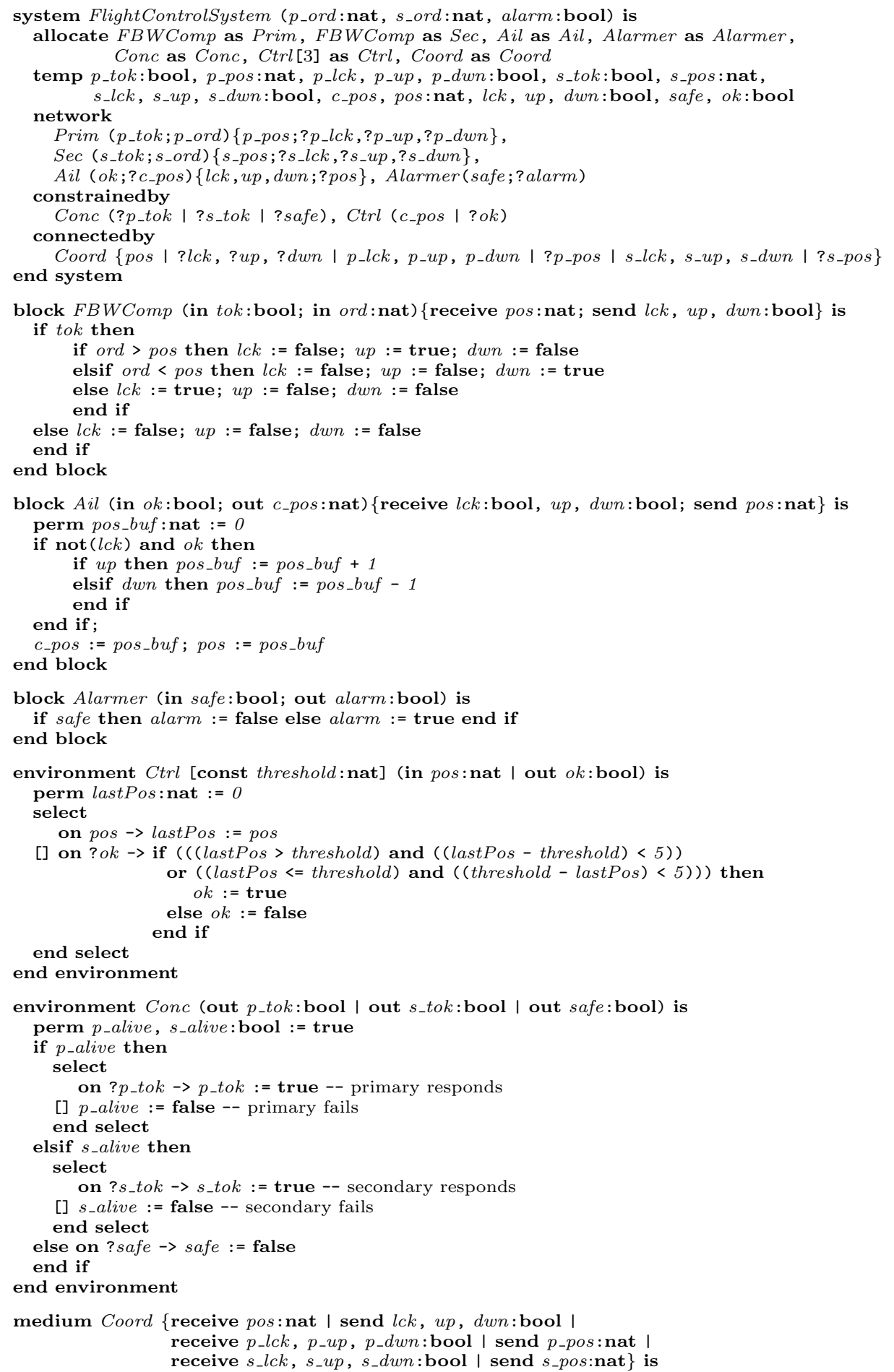




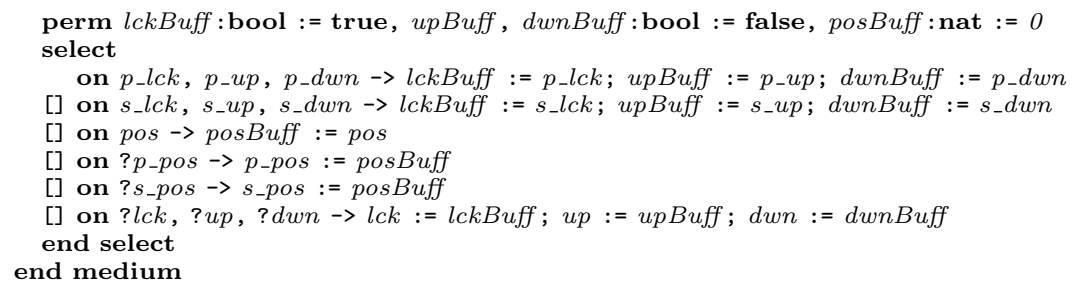

LTS generation. The GRL model has been translated into an LNT specification using the GRL2LNT tool, yielding a code that is 2.5 times larger than the input GRL model. Using CADP [11], the LTS of the model has been generated (2, 653 states, 7,406 transitions) then reduced modulo branching bisimulation (5 states, 1, 287 transitions), naturals being represented on 8 bits. This apparently small LTS size can be explained by the following facts. Different states represent different values of permanent variables whereas inputs and outputs only appear on transitions (temporary variables are not stored but only used in intermediate computations). Only variables $p_{-}$ord, s_ord, and alarm are visible on the LTS. Other variables (17 inputs and outputs) are hidden and thus do not occur in transition labels. Environment $C t r l$ constraints the range of possible positions to which Ail can move, thus drastically reducing the LTS. Reduction modulo branching bisimulation also helps in keeping the LTS small.

\section{Conclusion and Future Work}

We gave an overview of GRL, a new language with user-friendly syntax and formal semantics for modeling GALS systems, intended to enhance their design process. GRL combines synchronous features of dataflow languages and asynchronous features of process algebras, and makes possible a versatile, modular description of synchronous subsystems, environment constraints, and asynchronous communications. We designed GRL initially as a pivot language intended to facilitate the connection of industrial environments for designing PLCs (Programmable Logic Controllers) to formal verification tools. However, the language appears to be sufficiently expressive and general-purpose to model a wide range of GALS architectures (possibly nondeterministic), implemented on single or distributed platforms, and involving point-to-point or multi-point communications. Moreover, its user-friendly syntax and abstraction level, which is close to the dataflow model used in industry, makes GRL easier to learn and employ than a full-fledged process algebraic language like LNT.

GRL can independently be connected to verification frameworks based on either the synchronous or the asynchronous paradigms. The language is currently equipped with an automated translator to LNT, which makes possible the analysis of GRL descriptions using the rich functionalities of the CADP toolbox (e.g., simulation, verification, performance evaluation), focusing on the asynchronous behaviour of the GALS. GRL and the GRL2LNT translator start 
to be used in the Bluesky industrial project ${ }^{3}$, which addresses the validation of PLC networks. After a positive feedback received from our industrial partners, we are investigating an automated connection between their PLC design software (based on function block diagrams) and GRL, which would provide a complete analysis chain having CADP as verification back-end. We also develop reusable GRL programs describing basic function blocks and mediums corresponding to communication protocols used in PLC networks.

We plan to continue our work by applying equivalence checking and model checking techniques to industrial GALS systems described in GRL. Hardware/software co-simulation is also possible using the EXEC/CAESAR framework [14] of CADP, which enables the $\mathrm{C}$ code generated from a GRL description to be integrated with a physical platform. We also plan to investigate the connection of GRL to verification frameworks based on the synchronous paradigm to analyse the behaviour of individual blocks corresponding to synchronous subsystems.

\section{References}

1. K. Bae, P.C. Ölveczky, and J. Meseguer. Definition, semantics, and analysis of multirate synchronous aadl. In Proc. of FM. Springer, 2014.

2. G. Berry and G. Gonthier. The ESTEREL synchronous programming language: design, semantics, implementation. Science of Computer Programming, 19(2):87152, November 1992.

3. G. Berry, S. Ramesh, and R.K. Shyamasundar. Communicating reactive processes. In Proc. of POPL, pages 85-98. ACM Press, 1993.

4. G. Berry and E. Sentovich. Multiclock Esterel. In Proc. of CHARME, volume 2144 of $L N C S$, pages 110-125. Springer, 2001.

5. T. Bultan. Action language: A specification language for model checking reactive systems. In Proc. of ICSE. ACM, 2000.

6. J. Carlsson, K. Palmkvist, and L. Wanhammar. Synchronous design flow for Globally Asynchronous Locally Synchronous systems. In Proc. of ICC. WSEAS, 2006.

7. D. Champelovier, X. Clerc, H. Garavel, Y. Guerte, C. McKinty, V. Powazny, F. Lang, W. Serwe, and G. Smeding. Reference manual of the LOTOS NT to LOTOS translator (version 5.4). INRIA/VASY, September 2011.

8. N. Coste, H. Hermanns, E. Lantreibecq, and W. Serwe. Towards Performance Prediction of Compositional Models in Industrial GALS Designs. In Proc. of $C A V$, LNCS. Springer, 2009.

9. F. Doucet, M. Menarini, I.H. Krüger, R.K. Gupta, and J.-P. Talpin. A verification approach for GALS integration of synchronous components. Electr. Notes Theor. Comput. Sci., 146(2):105-131, 2006.

10. H. Garavel, F. Lang, and R. Mateescu. Compiler Construction Using LOTOS NT. In Proc. of CC, volume 2304 of $L N C S$. Springer, 2002.

11. H. Garavel, F. Lang, R. Mateescu, and W. Serwe. CADP 2011: A Toolbox for the Construction and Analysis of Distributed Processes. STTT, 15(2):89-107, 2013.

12. H. Garavel, G. Salaun, and W. Serwe. On the Semantics of Communicating Hardware Processes and their Translation into LOTOS for the Verification of Asynchronous Circuits with CADP. Science of Computer Programming, 2009.

\footnotetext{
${ }^{3}$ www.minalogic.com
} 
13. H. Garavel and D. Thivolle. Verification of GALS Systems by Combining Synchronous Languages and Process Calculi. In Proc. of SPIN, volume 5578 of LNCS, pages 241-260. Springer, 2009.

14. H. Garavel, C. Viho, and M. Zendri. System design of a CC-NUMA multiprocessor architecture using formal specification, model-checking, co-simulation, and test generation. STTT, 3(3):314-331, 2001.

15. A. Girault and C. Ménier. Automatic Production of Globally Asynchronous Locally Synchronous Systems. In Proc. of EMSOFT, volume 2491 of LNCS, pages 266-281. Springer, 2002.

16. H. Günther, S. Milius, and O. Möller. On the Formal Verification of Systems of Synchronous Software Components. In Proc. of SAFECOMP, volume 7612 of LNCS. Springer, 2012.

17. D. Harel. Statecharts: A visual formalism for complex systems. Science of computer programming, 8(3):231-274, 1987.

18. C.L. Heitmeyer, R.D. Jeffords, and B.G. Labaw. Automated consistency checking of requirements specifications. ACM Trans. on Software Engineering and Methodology, 5(3):231-261, 1996.

19. C.A.R. Hoare. Communicating Sequential Processes. Communications of the ACM, 21(8):666-677, August 1978.

20. G.J. Holzmann. The model checker SPIN. IEEE Transactions on Software Engineering, 23(5), 1997.

21. F. Jebali, F. Lang, and R. Mateescu. GRL: A Specification Language for Globally Asynchronous Locally Synchronous Systems. Research Report 8527, Inria, April 2014. Available online at http://hal.inria.fr/hal-00983711.

22. E. Lantreibecq and W. Serwe. Model Checking and Co-simulation of a Dynamic Task Dispatcher Circuit using CADP. In Proc. of FMICS, 2011.

23. N.G. Leveson, M.P.E. Heimdahl, H. Hildreth, and J.D. Reese. Requirements specification for process-control systems. IEEE Trans. on Software Engineering, 20(9):684-707, 1994.

24. A. Malik, Z. Salcic, P.S. Roop, and A. Girault. SystemJ: A GALS language for system level design. Comput. Lang. Syst. Struct., 36(4):317-344, December 2010.

25. S. Miller, E. Anderson, L. Wagner, M. Whalen, and M. Heimdahl. Formal verification of flight critical software. In Proc. of the AIAA Guidance, Navigation and Control Conference and Exhibit, 2005.

26. M.R. Mousavi, P. Le Guernic, J.-P. Talpin, S.K. Shukla, and T. Basten. Modeling and Validating Globally Asynchronous Design in Synchronous Frameworks. In Proc. of DATE. IEEE Computer Society, 2004.

27. F. Moutinho and L. Gomes. State space generation for Petri nets-based GALS systems. In Proc. of ICIT, 2012.

28. J. Muttersbach, T. Villiger, and W. Fichtner. Practical design of globallyasynchronous locally-synchronous systems. In Proc. of the International Symposium on Advanced Research in Asynchronous Circuits and Systems, 2000.

29. S Ramesh. Communicating reactive state machines: Design, model and implementation. In IFAC Workshop on Distributed Computer Control Systems, 1998.

30. M. Singh and M. Theobald. Generalized latency-insensitive systems for single-clock and multi-clock architectures. In Proc. of DATE, volume 2. IEEE, 2004.

31. L.H. Yoong, G. Shaw, P.S. Roop, and Z. Salcic. Synthesizing Globally Asynchronous Locally Synchronous Systems With IEC 61499. IEEE Transactions on Systems, Man, and Cybernetics, Part C, 42(6):1465-1477, 2012. 